\title{
Potencialización de la actividad antifúngica de propóleos mexicanos con la adición de chalconas sintetizadas
}

\author{
Potentialization of the Mexican propolis antifungal activity by the addition of synthetic chalcones
}

\begin{abstract}
Betsabé Rodríguez Pérez', José Guillermo Penieres Carrillo', María Margarita Canales Martínez², Ricardo Alfredo Luna Mora', Tonatiuh Alejandro Cruz Sánchez ${ }^{1}$

Facultad de Estudios Superiores Cuautitlán. Universidad Nacional Autónoma de México. Carretera Cuautitlán-Teoloyucan Km. 2.5, San Sebastián Xhala, Cuautitlán Izcalli, Estado de México, México. C.P. 54714.

2 Facultad de Estudios Superiores Iztacala, Universidad Nacional Autónoma de México. Avenida de los Barrios Número 1, Colonia Los Reyes Iztacala Tlalnepantla, Estado de México. C.P. 54090.
\end{abstract}

\section{RESUMEN}

Actualmente muchos propóleos presentan un gran valor socioeconómico importante, debido a las diversas actividades biológicas que presentan, que son producidas por los componentes químicos existentes en ellos. Sin embargo, no todos los propóleos llegan a presentar actividad biológica considerable, ya que sus componentes no la propician y esto puede mejorarse con la adición de precursores de flavonoides como las chalconas.

En el presente trabajo, se informa acerca de la evaluación en la potencialización de la actividad antifúngica contra Candida albicans y Aspergillus flavus de dos propóleos mexicanos, uno con buena actividad antimicótica (propóleo del Estado de México) y otro con baja actividad (propóleo del Estado de Guanajuato), mediante la adición a ellos de las chalconas 1,3-difenilpropil-2E-1-ona (PLCR-1), 1-(3,4-dihidroxifenil)-4-(2,4,6-trihidroxifenil)prop-2E-en-1ona (PLCR-2) y 1-(3,4-dihidroxifenil)-4-(2,4-dihidroxifenil) prop-2E-en-1-ona (PLCR-3), en diferentes concentraciones de cada una y en combinaciones entre ellas, mediante la técnica de difusión en disco, siendo la chalcona PLCR-2 la que presentó los mejores resultados

Palabras clave: propóleos mexicanos, actividad antifúngica, chalconas, potencialización.

\section{ABSTRACT}

Many propolis are considered of great socioeconomic value due to their various biological activities, which are produced by the chemical components present in them. However, not all propolis come to exhibit considerably biological activity, since its components don't propitiate it but this can be improved with the addition of flavonoids precursors such as chalcones. In the present work we report the evaluation in the antifungal activity potentialization against Candida albicans and Aspergillus flavus of two Mexican propolis, one with good antifungal activity (propolis from the state of Mexico) and the other with low activity (propolis from the state of Guanajuato). We added chalcones: 1,3-diphenylpropyl-2E-1-one (PLCR-1), 1-(3,4-dihydroxyphenyl)-4-(2,4,6-trihydroxyphenyl) prop-2E-en-1-one (PLCR-2) and 1-(3,4-dihydroxyphenyl)-
4-(2,4-dihydroxyphenyl)prop-2E-en-1-one (PLCR-3) each at different concentrations and combinations between them, by means of the disc diffusion technique. Our results indicate that chalcone PLCR-2 presents the best results.

Keywords: Mexican propolis, antifungal activity, chalcones, potentialization.

\section{INTRODUCCIÓN}

El aumento en la prevalencia de las micosis, la aparición de cepas fúngicas resistentes a los antifúngicos del tipo azoles, fármacos que son el pilar de la terapia antifúngica actual (Bader et al., 2015) y los efectos secundarios que provocan éstos últimos en los pacientes, se ha observado cada vez más a nivel mundial; esto ha generado la búsqueda y desarrollo de nuevos compuestos que cumplan con los requerimientos de un antifúngico ideal (Quintero-Mora et al., 2008). Los extractos etanólicos de propóleos poseen actividad fungicida y fungistática en diferentes especies de levaduras y hongos filamentosos, propiedad de importancia por ser una opción terapéutica económica y poco tóxica respecto a los antimicóticos tradicionales (Joya y Bastida-Pacheco, 2017). La inclinación del ser humano hacia el aprovechamiento de los productos naturales, se ha convertido en la actualidad en una opción insuperable para ser empleados en el tratamiento de diversas enfermedades (Londoño-Orozco et al., 2008).

Los propóleos, son una resina elaborada por las abejas Apis mellifera a partir de exudados de la vegetación circundante a la colmena y que la utilizan con fines desinfectantes y de embalsamiento de cadáveres de invasores en ella (AI et al., 2016; Bankova, 2005; Silva-Carvalho et al., 2015). Se han reportado e identificado hasta más de 300 compuestos químicos en algunos propóleos en todo el mundo, que ha apoyado a la investigación y aplicación en los potenciales benéficos para la salud (Bankova et al., 2016; Hernández-Zarate et al., 2017; Lotti et al., 2010). La presencia de los compuestos y su naturaleza química, es importante para que los propóleos lleguen a presentar actividad biológica, pero también el efecto sinérgico de estos compuestos es esencial para las actividades biológicas como la antimicrobiana, antifúngica, antiviral, antioxidante, entre otras (Bankova et al., 2016; Miguel et al., 2014).

*Autor para correspondencia: Betsabé Rodríguez Pérez Correo electrónico: berope380@hotmail.com Recibido: 14 de noviembre de 2018 Aceptado: 23 de marzo de 2019 
Por otro lado, las chalconas son metabolitos secundarios ampliamente distribuidos en la naturaleza y son precursores en la biosíntesis de flavonoides (Sapra et al., 2016). Su estructura química (Figura 1) presenta un anillo aromático en cada extremo de una cadena de 3 átomos de carbono con un sistema carbonílico a, $\beta$-insaturado (1,3-difenilprop-2E-en1-ona) (Blanco et al., 2016).

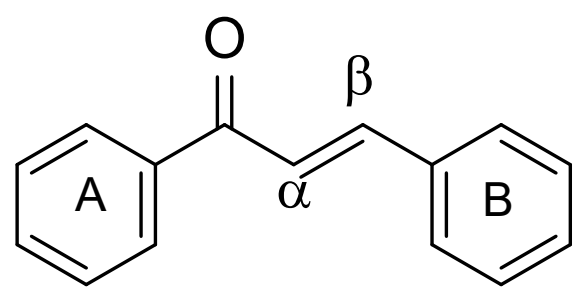

Figura 1. Estructura química de una chalcona (1,3-difenilprop-2E-en1-ona).

Figure 1. Chemical structure of a chalcone (1,3-diphenylprop-2E-en-1-one).

Este sistema carbonílico, la presencia y las posiciones de sustituyentes químicos, principalmente grupos hidroxilo, determinan el amplio rango de actividades biológicas y farmacológicas, considerándose compuestos con importantes potencialidades terapéuticas (Díaz-Tielas et al., 2016; Mahapatra y Bharti, 2016; Singh et al., 2014). Además, son compuestos que se pueden obtener en el laboratorio a partir de la síntesis química de condensación de Claisen-Schmidt entre la acetofenona o derivados de ella, con un aldehído aromático, mediante catálisis ácida o básica, seguido de una reacción de deshidratación (Figura 2) (Chetana et al., 2009).

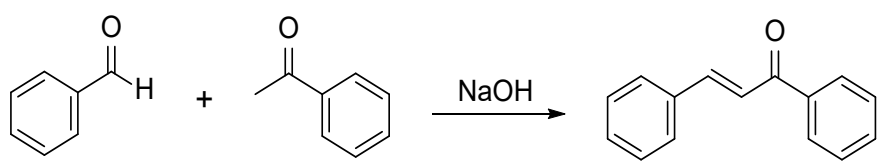

Figura 2. Síntesis general de chalconas.

Figure 2. General synthesis of chalcones.

Por lo tanto, el objetivo de esta investigación fue evaluar la potencialización de la actividad antifúngica contra Candida albicans y Aspergillus flavus de dos propóleos mexicanos, uno con buena actividad antimicótica (propóleo del Estado de México) y otro con baja actividad (propóleo del Estado de Guanajuato), mediante la adición a ellos de chalconas sintetizadas, en diferentes concentraciones de cada una y en combinaciones entre ellas.

\section{MATERIALES Y MÉTODOS Productos químicos}

Los reactivos y solventes utilizados en este estudio fueron obtenidos de Sigma-Aldrich México: benzaldehído y sus derivados (\# 1050905), acetofenona y sus derivados (\# 42163), hidróxido de sodio (\# 795429), ácido clorhídrico (\# 320331), hexano (\# 296090), acetato de etilo (\# 270989), alcohol etílico (\# 792780), reactivo de Folin-Ciocalteu (\# F9252), tricloruro de aluminio (\# 449598), metanol (\#
179957), acetonitrilo (\# 34998), ácido fórmico (\# F0507). Los estándares de flavonoides de ácido gálico (\# 91215) y quercetina (\# 1592409) fueron adquiridos de Sigma-Aldrich México. Los medios de cultivo fueron adquiridos en Becton Dickinson México.

\section{Recolección de los propóleos en greña}

Se obtuvieron dos propóleos en greña o en bruto de la cosecha de verano (septiembre de 2015). Un propóleo proveniente del apiario de la Facultad de Estudios Superiores Cuautitlán (Estado de México) con localización geográfica al norte $19^{\circ} 41^{\prime}$ y al oeste $99^{\circ} 11^{\prime}$, con altura de $2260 \mathrm{msnm}$ y recolectado utilizando mallas plásticas. El otro propóleo corresponde al municipio de San José Iturbide, Guanajuato con coordenadas al norte $21^{\circ} 07^{\prime} y$ al oeste $100^{\circ} 32^{\prime}$, con altura de 2100 msnm y recolectado por el método de raspado. A las muestras se les eliminaron las impurezas, previo a las pruebas organolépticas.

\section{Microorganismos}

Los hongos utilizados para este estudio fueron una cepa de referencia de Candida albicans (ATCC 14065) y una de Aspergillus flavus proveniente de un aislamiento de campo a partir de granos de maíz, los cuales fueron seleccionados por constituir importantes microorganismos patógenos de humanos y animales, y este hongo filamentoso tiene afinidad por un amplio rango de huéspedes incluyendo el ser humano, los animales y las plantas, además de ser el patógeno que está involucrado con mayor frecuencia en la producción de aflatoxinas. Ambos microorganismos fueron proporcionados por el laboratorio de Bioprospección Microbiológica de la Facultad de Estudios Superiores Cuautitlán, UNAM.

\section{Antifúngicos}

Se utilizaron antifúngicos comerciales de ketoconazol de $50 \mu \mathrm{g}$ (Bio-Rad catálogo 62866) y de voriconazol de $1 \mu \mathrm{g}$ (Bio-Rad catálogo 62803).

\section{Sensidiscos}

Se utilizaron discos de papel filtro de $6 \mathrm{~mm}$ de diámetro (Whatman No. 5) previamente esterilizados.

\section{Preparación del extracto etanólico de propóleo (EEP)}

Para la obtención del extracto etanólico se utilizó la siguiente metodología: se pesó una cantidad de $50 \mathrm{~g}$ de los propóleos en greña, previa limpieza, se añadió alcohol etílico al 70 \% en una proporción 3:1 de disolvente:propóleo, para someterlas a extracción en un Ultrasonicador (Branson, CPX1800H, Danbury, Estados Unidos). Posteriormente, se separó por filtración utilizando un embudo Buchner y el filtrado resultante se concentró utilizando un Rotovapor (Science MED, SM100-PRO, Finlandia) y se pasó a un frasco ámbar utilizando la mínima cantidad de alcohol etílico al 70 $\%$. Se dejó a sequedad utilizando una bomba de vacío para eliminar los residuos de etanol. El extracto seco se pesó y almacenó protegido de la luz y en refrigeración a $4{ }^{\circ} \mathrm{C}$ hasta su utilización (NOM-003-SAG/GAN-2017; Trusheva et al., 2007). 


\section{Síntesis de chalconas}

Para la síntesis de chalconas, se realizó la reacción de condensación de Claisen-Schmidt entre el benzaldehído y la acetofenona correspondiente, a una concentración de 0.01 $M$, respectivamente. La mezcla se colocó en un matraz bola de $250 \mathrm{~mL}$ de fondo redondo equipado con un agitador magnético. Posteriormente, se agregó gota a gota sobre la mezcla de reacción, $10 \mathrm{~mL}$ de una solución de $\mathrm{NaOH}(0.1$ M) por un lapso de 30 min manteniendo la temperatura de reacción entre $20^{\circ} \mathrm{C}$ y $25^{\circ} \mathrm{C}$, utilizando un baño de hielo. La mezcla de reacción se dejó en agitación constante por $5 \mathrm{~h}$ y a continuación se neutralizó con $\mathrm{HCl} 0.1 \mathrm{~N}$, se dejó enfriar hasta la formación de un precipitado. El precipitado fue filtrado por gravedad y se dejó secar a temperatura ambiente. El producto principal fue purificado utilizando cromatografía en columna (sílica gel y hexano:acetato de etilo en proporción de 8:2) para la obtención de las chalconas puras (Chetana et al., 2009; Hormaza et al., 2009).

\section{Cuantificación de compuestos fenólicos y flavonoides}

Las determinaciones se realizaron conforme a lo establecido en la NOM-003-SAG/GAN-2017. Para medir la cantidad de compuestos fenólicos se utilizó el método de Folin-Ciocalteu (FC) con base a una reacción colorimétrica de óxido-reducción, utilizando una longitud de onda de 765 nm y empleando ácido gálico como solución estándar. Se utilizó el método colorimétrico del tricloruro aluminio $\left(\mathrm{AlCl}_{3}\right)$ para la cuantificación de flavonoides, utilizando quercetina como estándar de referencia y se determinó la absorbancia utilizando una longitud de onda de $415 \mathrm{~nm}$. Las lecturas de absorbancia se realizaron utilizando un espectrofotómetro de absorción UV-VIS (DLAB, SP-UV1000, China). Los resultados se interpolaron en la gráfica de la curva de calibración. Los resultados de ambas determinaciones se expresaron en porcentaje (NOM-003-SAG/GAN-2017; Rodríguez, 2015).

\section{Cromatografía de Gases acoplada Espectroscopía de Masas (CG-EM)}

El análisis de los extractos etanólicos fue realizado en un cromatógrafo de gases (Agilent Technologies 6850) acoplado a un espectrómetro de masas (Agilent Technologies, 5975C). La columna capilar utilizada fue HP-5MS (30 m x $0.25 \mathrm{~mm}$ ) y con grosor de película de $0.25 \mathrm{~mm}$, utilizando helio como gas acarreador. El tipo de inyección fue en modo Split y el volumen de inyección de $1 \mu \mathrm{L}$. Las condiciones de separación utilizadas fueron al inicio, una temperatura de 70 ${ }^{\circ} \mathrm{C}$ por dos min con dos incrementos de rampa, el primero de $20^{\circ} \mathrm{C}$ por min hasta alcanzar los $230^{\circ} \mathrm{C}$ y el segundo fue de $8{ }^{\circ} \mathrm{C}$ por min hasta llegar a los $280^{\circ} \mathrm{C}$ manteniéndose por $5 \mathrm{~min}$. El tiempo total de análisis fue $21.25 \mathrm{~min}$. El rango de masa detectado fue de $35-750 \mathrm{~m} / \mathrm{z}$, la muestra fue ionizada por impacto electrónico a 70 eV y la temperatura alcanzada por la fuente de ionización fue de $230^{\circ} \mathrm{C}$. La identificación de los compuestos fue llevada a cabo por comparación con la biblioteca de la base de datos NIST versión 8.0. (Rivera-Yáñez et al., 2017).

\section{Cromatografía de Líquidos de Alta Resolución (HPLC- DAD)}

Se utilizaron $30 \mu \mathrm{L}$ del extracto de propóleo, se inyectó a una concentración de $3 \mathrm{mg} / \mathrm{mL}$ en un equipo de HPLC Hewlett-Packard HP modelo 1100 (Hewlett-Packard, Wilmington $\mathrm{DE}, \mathrm{EU})$ con un detector de matriz de diodos (DAD) 1100 operado con ChemStation A0903 con los siguientes parámetros: separación isocrática usando una fase móvil metanol:acetonitrilo:agua (25:25:50) acidificada con ácido fórmico (1\%) durante 60 min; columna Discovery C-18 $(250 \times 4.6 \mathrm{~mm})$, a 269 bar de presión y un rango de temperatura de $23{ }^{\circ} \mathrm{C}$; velocidad de flujo, $1 \mathrm{~mL} / \mathrm{min}$; matriz de detectores de diodos con ajuste del detector a $260 \mathrm{~nm}$; y escaneo completo de $200-400 \mathrm{~nm}$. Los constituyentes se identificaron con base a una comparación del tiempo de retención y el espectro UV con los estándares (Rivera-Yañez et al., 2018).

\section{Evaluación de la actividad antifúngica}

Preparación de los sensidiscos. Se impregnaron discos estériles de papel filtro de $6 \mathrm{~mm}$ de diámetro (Whatman No. 5) con $6 \mathrm{mg} / 10 \mu \mathrm{L}$ de los extractos de propóleos y la misma cantidad para las chalconas a evaluar, dejándose secar durante $24 \mathrm{~h}$. Como testigo negativo se utilizaron discos impregnados con alcohol etílico al $70 \%$. Como testigos positivos se utilizaron antifúngicos comerciales de ketoconazol de $50 \mu \mathrm{g}$ para C. albicans y de voriconazol de $1 \mu \mathrm{g}$ para $A$. flavus (CLSI/M44-A2, 2009; NOM-003-SAG/GAN-2017).

Candida albicans (método de difusión en disco). Se inoculó a la levadura en caldo Dextrosa Sabouraud (BD Dioxon), se incubó a $35^{\circ} \mathrm{C}$ por $48 \mathrm{~h}$, se realizaron las pruebas de identificación y se ajustó al tubo 0.5 de la escala del nefelómetro de MacFarland utilizando un espectrofotómetro, con una absorbancia entre 0.08-0.12 a una longitud de onda de $625 \mathrm{~nm}$. A partir del inóculo preparado, se realizó un sembrado masivo sobre placas de agar Mueller-Hinton suplementado con $2 \%$ de glucosa y $0.5 \mu \mathrm{g} / \mathrm{mL}$ de azul de metileno (BD Dioxon). Se colocaron los discos impregnados con las muestras a evaluar, los testigos negativos y positivos. Se incubaron por $24 \mathrm{~h}$ a $37^{\circ} \mathrm{C}$ y se midieron los halos de inhibición y a las 48 h se midió nuevamente el diámetro de los halos. Los resultados se expresaron como el promedio del diámetro de los halos de inhibición para cada tratamiento (CLSI/M44-A2, 2009; NOM-003-SAG/GAN-2017).

Aspergillus flavus (Método de inhibición del crecimiento radial). El hongo filamentoso fue sembrado en placas de agar SDA (BD Dioxon) que fueron incubadas a 28 'C hasta que el desarrollo se apreció en toda la superficie del agar. Se tomó un inóculo de $5 \mathrm{~mm}$ de diámetro del hongo y se resembró en agar SDA (BD Dioxon), colocando los discos impregnados con las muestras a evaluar, los testigos negativos y positivos. Se incubaron a $28{ }^{\circ} \mathrm{C}$ por $72 \mathrm{~h}$, hasta que la superficie del agar estuvo cubierta por el micelio. Se observó la formación de zonas de inhibición con formas de media luna. Los resultados se expresaron como el promedio del 
diámetro de los halos de inhibición para cada tratamiento (CLSI M44-A2; Londoño, 2010).

\section{Ensayo de potencialización entre EEP y chalconas}

Se realizaron los ensayos de potencialización después de evaluar la actividad antifúngica de manera individual, de los EEP y de las chalconas. Se realizaron modificaciones a las metodologías utilizadas para los ensayos de evaluación de sinergia entre EEP y antimicrobianos (Mirzoeva et al., 1997; Stepanović, et al., 2003). Para este ensayo se realizó el siguiente procedimiento: se prepararon diferentes concentraciones de EEP de 1 a $5 \mathrm{mg} / \mathrm{mL}$ y una concentración de $1 \mathrm{mg} / \mathrm{mL}$ para cada chalcona. Se realizaron los cálculos químicos necesarios para tener cada una de las siguientes combinaciones entre cada una de las chalcona con cada EEP: combinación A: 1 parte de chalcona/1 parte de EEP (1:1); combinación B: 1 parte de chalcona/2 partes de EEP (1:2); combinación C: 1 parte de chalcona/3 partes de EEP (1:3); combinación D: 1 parte de chalcona/4 partes de EEP (1:4) y combinación E: 1 parte de chalcona/5 partes de EEP (1:5). Se impregnaron discos estériles con $10 \mu \mathrm{L}$ de cada combinación y se realizó el procedimiento para evaluar la actividad antifúngica como se describió con anterioridad.

\section{Análisis estadístico}

Los datos obtenidos se analizaron mediante la prueba estadística de Análisis de varianza (ANOVA) empleando un nivel de significancia de $\mathrm{P}<0.05$ utilizando el Programa Estadístico GraphPad Prism 7.04.

\section{RESULTADOS Y DISCUSIÓN}

\section{Síntesis de chalconas}

Las tres chalconas sintetizadas mediante la reacción de condensación de Claisen-Schmidt fueron: 1,3-difenilpropil-2E-1-ona (PLCR-1) y dos análogos hidroxisustituidos en los anillos $A$ y B: 1-(3,4-dihidroxifenil)-4-(2,4,6-trihidroxifenil) prop-2E-en-1-ona (PLCR-2) y 1-(3,4-dihidroxifenil)-4-(2,4dihidroxifenil)prop-2E-en-1-ona (PLCR-3), las estructuras se muestran en la Figura 3.

El compuesto PLCR-1 presentó un aspecto de cristales amarillos con rendimiento del $88 \%$; el compuesto PLCR-2, un aspecto de polvo café con rendimiento del $71 \%$ y el compuesto PLCR-3, un polvo rojo con rendimiento del $96 \%$. Los porcentajes de rendimiento presentan un valor promedio de $85 \%$, siendo satisfactorios debido a que es una metodología sencilla, ecológica, económica y es un tema de gran interés en la actualidad (Ramírez et al., 2012).

\section{Recolección de propóleos y especificaciones físicas de los propóleos}

Se evaluó el color, el aroma, el sabor y la consistencia. Para la evaluación del color se utilizó un microscopio estereoscópico para observar detenidamente las tonalidades predominantes en cada muestra. El aroma se describió como: resinoso (madera) o balsámico (cera). El sabor se identificó como dulce, amargo, picante o insípido. La consistencia se determinó al tocar la muestra con los dedos, describiéndola como maleable o rígida (Lozina et al., 2010; NOM-003-SAG/ GAN-2017).
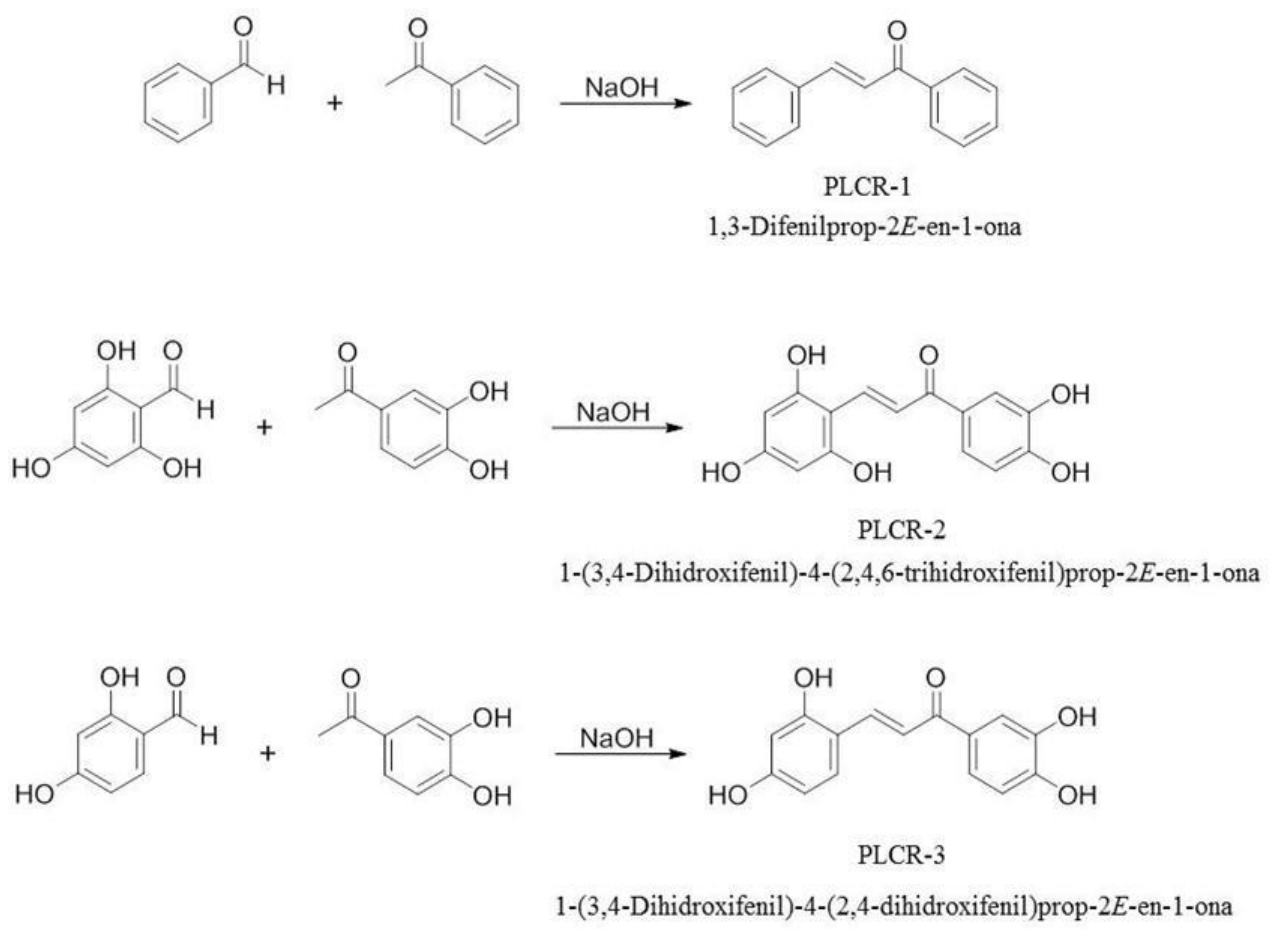

Figura 3. Síntesis general de chalconas PLCR 1-3.

Figure 3. General synthesis of chalcones PLCR 1-3. 
La normatividad mexicana establece que la producción y la recolección de los propóleos debe realizarse utilizando mallas de plástico para facilitar su obtención y evitar la manipulación directa con el fin de garantizar que su composición química no se afecte. El propóleo del Estado de México (EM) se produjo bajo lo establecido en la normatividad mexicana, se obtuvo un propóleo de aspecto granulado y brilloso, que se compara con lo reportado por Viloria et al (2012). El propóleo de Guanajuato (G) se recolectó por el método de raspado presentando aspecto de trozos irregulares y opacos como lo reporta Bucio et al. (2016).

Las características físicas de los propóleos están relacionadas con la fuente vegetal de donde las abejas recolectan la resina. El color que prevaleció en ambas muestras fue marrón con tintes verdes y se percibió un sabor amargo; el aroma del propóleo del EM fue balsámico, relacionado con la presencia de aceites esenciales de Eucaliptus sp. y Pinus sp. (Londoño, 2010; Sosa-López et al., 2017). Para la muestra de $\mathrm{G}$, el aroma fue resinoso (olor a madera) por los aceites esenciales del mezquite (Prosopis glandulosa Torr.), principal especie establecida en la proximidad del apiario (GarcíaAndrade et al., 2013).

\section{Composición química de los extractos de propóleos}

La composición química de los propóleos es un rico conjunto de compuestos fenólicos y flavonoides que determinan su bioactividad y depende del origen botánico, la época y del método de recolección (Bankova, 2009). Los resultados de la composición química de los propóleos se muestran en la Tabla 1. Ambos propóleos cumplen con las especificaciones de calidad mexicana. Los compuestos detectados en la muestra del EM corresponden a los flavonoides, pinocembrina, kaempferol, crisina y quercetina, identificados en propóleos del mismo apiario como compuestos mayoritarios en otros estudios (Flores, 2018; González-Búrquez et al., 2018; Rodríguez, 2015); también se detectaron en menor cantidad, ácido palmítico y ácido oleico que están presentes en las ceras (Vargas-Sánchez et al., 2014) y en especies de álamos (Lugo et al., 2006), Eucaliptus sp (Londoño, 2010) y Pinus sp (Rasul et al., 2013). En el propóleo de G no se detectaron flavonoides, únicamente un terpeno, la ( $E$ )-a-Damascona; estos resultados concuerdan con los obtenidos por Rodríguez (2015), que se relaciona con factores como: el origen botánico que define la cantidad y calidad de los metabolitos; la época de recolección, pues existen reportes de la presencia únicamente de terpenos en propóleos producidos en el verano y otoño (Bankova et al., 2016); la obtención por la técnica de raspado, donde puede existir contaminación física por madera o pintura (Bucio et al., 2016), la exposición al calor y la manipulación de la resina, principalmente.

\section{Actividad antifúngica y ensayo de potencialización entre EEP y chalconas}

La actividad antifúngica es una de las propiedades fundamentales de los propóleos (Bankova et al., 2016), siendo de importancia el estudio de la composicion química.

Candida albicans. Se realizaron pruebas de identificación a partir del inóculo preparado (Figura 4A y 4B) y los resultados de la actividad antifúngica de las muestras estudiadas se muestran en la Tabla 2.

El ketoconazol es un antifúngico que inhibe la ruta biosintética del ergosterol, componente lipídico de la membrana fúngica y probado clínicamente (Tapia, 2005) se observó la sensibilidad de la levadura frente a este fármaco. Los EEP no inhibieron el crecimiento de la cepa de referencia, pero existen estudios previos que indican que propóleos obtenidos del mismo apiario en distintas épocas, presentan efecto fungicida y fungistático en cepas de referencia y muestras de aislamientos clínicos de C. albicans (QuinteroMora et al., 2008), por lo que es necesaria una evaluación utilizando este tipo de muestras para corroborar los resultados. La levadura presentó sensibilidad frente a las tres chalconas, sin diferencias significativas entre los halos de inhibición. Se ha reportado que la actividad antifúngica de las chalconas es por factores como: la presencia del grupo carbonilo $\alpha, \beta-$ insaturado; la posición y la cantidad de los grupos hidroxilo en los anillos aromáticos A y B (Kulkarni et al., 2009) y la sustitución de la cetona y el doble enlace $C=C$ (enona) que se une al grupo tiol de las glicoproteínas de la pared fúngica provocando la inhibición en la síntesis de 1,3- $\beta$-D-glucano

Tabla 1. Composición química de propóleos mexicanos identificados por CG-EM y HPLC-DAD.

Table 1. Chemical composition of Mexican propolis, identified by GC-MS and HPLC-DAD.

\begin{tabular}{|c|c|c|c|c|c|c|c|c|}
\hline \multirow[b]{2}{*}{ Muestra } & \multirow[b]{2}{*}{ \% Fenoles* } & \multirow{2}{*}{$\begin{array}{c}\% \\
\text { Flavonoides* }\end{array}$} & \multicolumn{3}{|c|}{ CG-EM } & \multicolumn{3}{|c|}{ HPLC-DAD } \\
\hline & & & $\mathrm{TR}$ (min) & $\begin{array}{c}\text { Abundancia } \\
\text { porcentual }\end{array}$ & $\begin{array}{l}\text { Compuestos } \\
\text { propuestos }\end{array}$ & $\begin{array}{c}\text { TR } \\
(\min ) \\
\end{array}$ & $\begin{array}{c}\text { Abundancia } \\
\text { porcentual }\end{array}$ & $\begin{array}{c}\text { Compuestos } \\
\text { propuestos }\end{array}$ \\
\hline \multirow{4}{*}{$\begin{array}{l}\text { EEP } \\
\text { Estado de } \\
\text { México }\end{array}$} & \multirow{4}{*}{$12.9 \pm 0.001^{\mathrm{a}}$} & \multirow{4}{*}{$5.4 \pm 0.001^{a}$} & 23.707 & 5.5 & Ácido palmítico & 5.410 & 18.7 & Pinocembrina \\
\hline & & & 25.094 & 2.9 & 2-Nonadecanona & 7.877 & 5.7 & Kaempferol \\
\hline & & & 25.801 & 3.5 & Ácido oleico & 8.837 & 0.9 & Quercetina \\
\hline & & & 29.594 & 68 & Pinocembrina & 15.424 & 7.0 & Crisina \\
\hline EEP Guanajuato & $7.5 \pm 0.002^{b}$ & $1.8 \pm 0.74^{b}$ & 29.66 & 18.8 & $(E)$-a-Damascona & & in compuesto & letectado \\
\hline
\end{tabular}

* Cada valor es la media \pm desviación estándar, $n=3$; Letras diferentes indican diferencias significativas $(p<0.05)$ 
Tabla 2. Actividad antifúngica de extractos de propóleos y potencialización de la actividad con chalconas PLCR-1-3 frente a Candida albicans ATCC 14053.

Table 2. Antifungal activity of propolis extracts and potentialization of their activity with PLCR-1-3 chalcones against Candida albicans ATCC 14053.

\begin{tabular}{|c|c|c|c|c|c|c|}
\hline & & \multicolumn{5}{|c|}{ Halo de inhibición (mm) } \\
\hline \multicolumn{3}{|l|}{ Ketoconazol } & \multicolumn{4}{|c|}{$24 \pm 1.0^{\mathrm{a}}$} \\
\hline \multicolumn{3}{|c|}{ Alcohol etílico al $70 \%$} & \multicolumn{4}{|l|}{0.0} \\
\hline \multicolumn{3}{|c|}{ Extracto del Estado de México (EM) } & \multicolumn{4}{|l|}{0.0} \\
\hline \multicolumn{3}{|c|}{ Extracto de Guanajuato (G) } & \multicolumn{4}{|l|}{0.0} \\
\hline \multicolumn{3}{|l|}{ PLCR-1 } & \multicolumn{4}{|c|}{$12.0 \pm 1.0^{\mathrm{b}}$} \\
\hline \multicolumn{3}{|l|}{ PLCR-2 } & \multicolumn{4}{|c|}{$9.3 \pm 1.5^{b}$} \\
\hline \multicolumn{3}{|l|}{ PLCR-3 } & \multicolumn{3}{|c|}{$11.3 \pm 0.6^{b}$} & \\
\hline \multicolumn{7}{|c|}{$\begin{array}{l}\text { Prueba de potencialización } \\
\text { Halo de inhibición }(\mathrm{mm})\end{array}$} \\
\hline \multirow{2}{*}{$\begin{array}{l}\text { Proporción } \\
\text { evaluada }\end{array}$} & PLCR-1 & & \multicolumn{2}{|l|}{ PLCR-2 } & \multicolumn{2}{|l|}{ PLCR-3 } \\
\hline & EM & G & EM & G & EM & G \\
\hline 1:1 & 0.0 & $7.0 \pm 1.7^{\mathrm{a}}$ & $10.3 \pm 0.6^{\mathrm{a}}$ & $11.3 \pm 1.2^{\mathrm{a}}$ & 0.0 & 0.0 \\
\hline $1: 2$ & 0.0 & $7.3 \pm 0.6^{\mathrm{a}}$ & 0.0 & 0.0 & $8.0 \pm 0.0$ & 0.0 \\
\hline $1: 3$ & $11.3 \pm 0.6^{\mathrm{a}}$ & $7.0 \pm 0.0^{\mathrm{a}}$ & 0.0 & 0.0 & 0.0 & 0.0 \\
\hline $1: 4$ & 0.0 & $7.0 \pm 0.0^{\mathrm{a}}$ & 0.0 & 0.0 & 0.0 & 0.0 \\
\hline 1:5 & $9.0 \pm 1.0^{b}$ & 0.0 & $15.7 \pm 0.6^{b}$ & $7.0 \pm 1.0^{\mathrm{b}}$ & 0.0 & 0.0 \\
\hline
\end{tabular}

Cada valor es la media \pm desviación estándar, $n=3$. Literales o letras diferentes indican diferencias significativas $(p<0.05)$

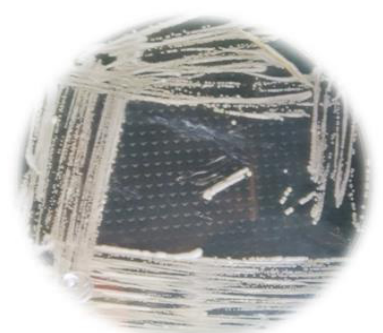

A

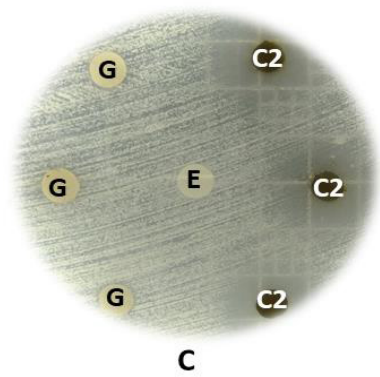

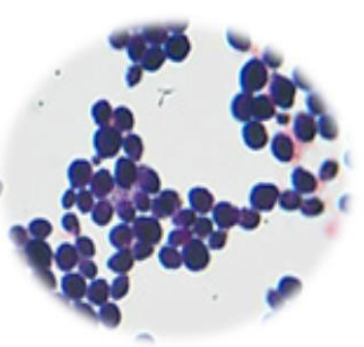

B

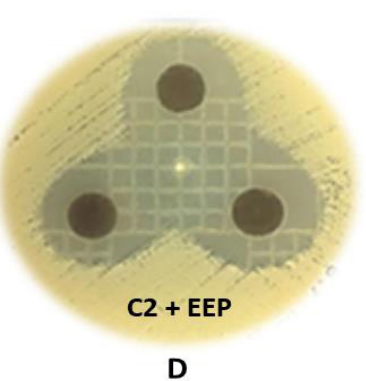

Figura 4. Evaluación de la sensibilidad de Candida albicans frente a las muestras estudiadas. A. Identificación macroscópica; B. Identificación microscópica; C. Comparación de la actividad antifúngica del extracto de propóleo de Guanajuato (G) y chalcona PLCR-2 (C2); D. Potencialización del efecto antifúngico de la mezcla del extracto de propóleo de Guanajuato y la chalcona PLCR-2.

Figure 4. Candida albicans sensitivity evaluation against studied samples. A. Macroscopic identification; B. Microscopic identification; C. Comparison between the antifungal activity of the propolis extract from Guanajuato (G) and PLRC-2 (C2); D. Potentialization of the antifungal effect of the Guanajuato propolis extract and PLCR-2 mixture. 
y de la glutatión-S-transferasa (Guerrero, 2016; Lopez et al., 2001; Sivakumar et al., 2009).

La evaluación de la potencialización de la actividad antifúngica se muestra en la Tabla 2. La muestra del EM puede presentar o no, el efecto inhibitorio en el crecimiento de C. albicans con base a la chalcona adicionada y la proporción evaluada; sin embargo, la adición de la chalcona PLCR-2 en proporción 1:5, provocó un mayor halo de inhibición (15.7 $\mathrm{mm}$ ) que el halo de inhibición de la chalcona por sí sola $(9.3$ $\mathrm{mm}$ ), considerando que el efecto no es particularmente por la chalcona ya que se encuentra en menor proporción.

La mezcla del EEP de G con la chalcona PLCR-3 presentó un antagonismo en todas las proporciones observando el crecimiento de la levadura. Con la PLCR-1, no existió diferencia significativa entre el diámetro de los halos de inhibición $(7.0 \mathrm{~mm})$ de las proporciones evaluadas, pero sí, con el halo de inhibición de la chalcona $(12 \mathrm{~mm})$; la adición de la chalcona PLCR-2 en la proporción 1:1 presentó mejor actividad antifúngica con un halo de inhibición mayor, al que genera la chalcona por sí sola (Figura 4D).

Con lo anterior se demostró que la adición de la chalcona PLCR-2 a los EEP, tiene mejor efecto de inhibición para C. albicans. Se puede proponer que la estructura química de esta chalcona, al presentar mayor número de sustituyentes hidroxilo provocan la alteración de los componentes de la pared celular y la potencialización de la actividad antifúngica, siendo necesarios estudios que comprueben esta hipótesis.

Aspergillus flavus. Se realizó la identificación del hongo por la evaluación de la morfología de las colonias de color verde con micelio amarillo, textura vellosa y el reverso de la colonia incoloro (Figura 5A). Utilizando la técnica de microcultivo de Riddel, se realizó la identificación de las características morfológicas utilizando las monografías de Larone para la observación microscópica de la cabeza conidial característica de este hongo (Figura 5B). (Cruz et al., 2014; Larone, 2011). Los resultados de la actividad antifúngica frente a este hongo se muestran en la tabla 3 . Se observó la sensibilidad del hongo frente al voriconazol, antifúngico triazólico de segunda generación utilizado para mejorar el pronóstico de las aspergilosis invasoras (Tapia, 2005) y también presentó a sensibilidad a la chalcona PLCR-2 (Figura 5C). Los resultados del ensayo de potencialización para las dos muestras de propóleos demostraron que la adición de la chalconas PLRC-2 y -3 en diferentes proporciones, incrementan la sensibilidad del hongo (Figura 5D).

Se observó que los dos hongos estudiados presentan una resistencia frente a los dos EEP y el A. flavus a las chalconas PLCR-1 y -3; existen estudios que reportan el mecanismo de acción de la resistencia de hongos a nuevos compuestos, como son: la existencia de barreras de permeabilidad o a sistemas de bombeo activo del compuesto al exterior, que impiden alcanzar la diana dentro de la célula; cambios en la interacción fármaco-diana debida a mutaciones; $y$, modificaciones en las enzimas de las vías metabólicas de la síntesis del ergosterol. Por lo anterior, es necesaria la realización de
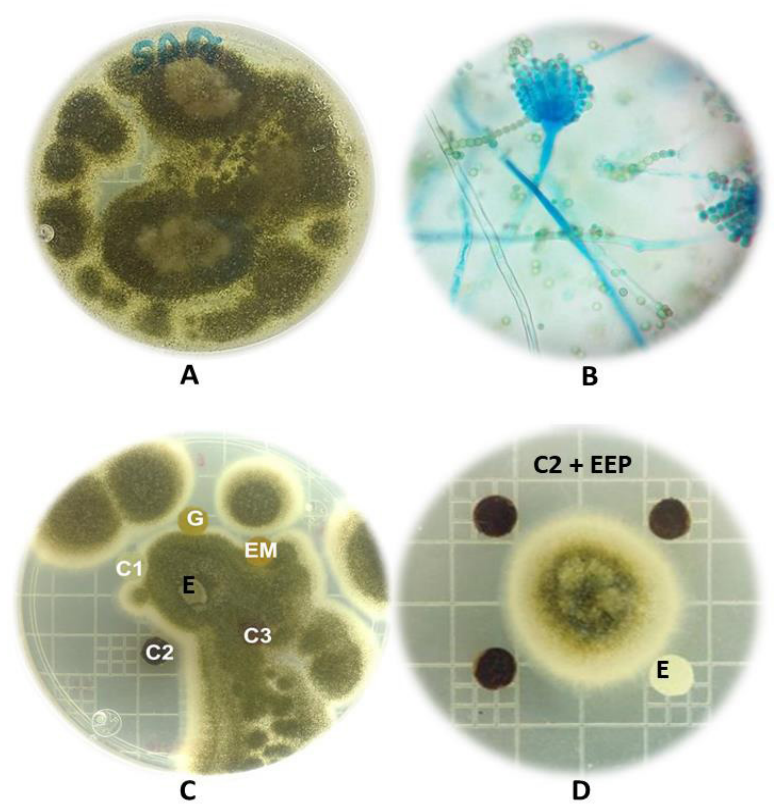

Figura 5. Evaluación de la sensibilidad de Aspergillus flavus frente a las muestras estudiadas. A. Identificación macroscopica; B. Identificación microscópica; C. Comparación de la actividad antifúngica de los extractos de propóleo del Estado de México (EM), Guanajuato (G), chalconas PLCR-1 (C1), PLCR-2 (C2) y PLCR-3 (C3), Etanol (E); D. Potencialización del efecto antifúngico de la mezcla del EEP y la chalcona PLCR-2. Se observan las medias lunas que indican la inhibición de crecimiento.

Figure 5. Sensitivity evaluation of Aspergillus flavus against the studied samples. A. Macroscopic identification; B. Microscopic identification; C. Comparison of the antifungal activity of the propolis ethanolic extracts from the State of Mexico,(SM), Guanajuato (G); PLCR-1 (C1), PLCR-2 (C2) and PLCR-3 (C3) chalcones, Ethyl alcohol (E); D. Potentialization of the antifungal effect for a PEE and PLCR-2 mixture.

pruebas más específicas a nivel molecular para poder elucidar la resistencia microbiológica y clínica como lo menciona Pfaller (2012), aunado a la preocupación en el uso masivo de azoles en la agricultura que podría favorecer la aparición de cepas resistentes y modificar la población de hongos que infectan al ser humano (Mellado et al., 2002).

Para el caso de los propóleos, en México se han realizado estudios de la composición química y del efecto antifúngico del propóleo del Estado de México proveniente de la FES Cuautitlán contra cepas de Candida albicans y por medio de microscopía electrónica se demostró la inhibición del tubo germinativo y cambios en la pared celular con sitios de gemación irregulares provocados por compuestos como el ácido cafeíco, flavonoides y ésteres fenólicos (Londoño et al., 2008). En un estudio realizado por Quintero et al. (2011), se concluyó que en C. albicans, se afectan los genes ADH1 y PIK1 que están relacionados con los cambios de la inhibición de la formación del tubo germinativo, la formación de blastoconidias y el cambio morfogenético de la levadura.

Se puede proponer que existe una sinergia entre los compuestos químicos de los propóleos y las chalconas que provocan la alteración de los componentes de la pared celular, siendo necesario la realización de estudios como microscopía electrónica, estudios genéticos y moleculares; 
Tabla 3. Prueba de inhibición del crecimiento radial de los extractos de propóleos y potencialización de la actividad de chalconas sintetizadas PLCR 1-3 frente a Aspergillus flavus.

Table 3. Propolis extracts inhibition of radial growth and activity potentialization of synthetic chalcones PLCR 1-3 against Aspergillus flavus.

\begin{tabular}{|c|c|c|c|c|c|c|}
\hline \multicolumn{7}{|c|}{ Halo de inhibición (mm) } \\
\hline \multicolumn{4}{|c|}{ Voriconazol $(1 \mu \mathrm{g})$} & \multicolumn{3}{|l|}{$18^{\mathrm{a}}$} \\
\hline \multicolumn{4}{|c|}{ Alcohol etílico al $70 \%$} & \multicolumn{3}{|c|}{0.0} \\
\hline \multicolumn{4}{|c|}{ Extracto de Estado de México (EM) } & \multicolumn{3}{|c|}{0.0} \\
\hline \multicolumn{4}{|c|}{ Extracto de Guanajuato (G) } & \multicolumn{3}{|c|}{0.0} \\
\hline \multicolumn{4}{|l|}{ PLCR-1 } & \multicolumn{3}{|c|}{0.0} \\
\hline \multicolumn{4}{|l|}{ PLCR-2 } & \multicolumn{3}{|c|}{$10^{b}$} \\
\hline \multicolumn{4}{|l|}{ PLCR-3 } & \multicolumn{3}{|c|}{0.0} \\
\hline \multicolumn{7}{|c|}{$\begin{array}{c}\text { Prueba de potencialización } \\
\text { Halo de inhibición (mm) }\end{array}$} \\
\hline \multirow{2}{*}{$\begin{array}{l}\text { Proporción } \\
\text { evaluada }\end{array}$} & \multicolumn{2}{|c|}{ PLCR-1 } & \multicolumn{2}{|c|}{ PLCR-2 } & \multicolumn{2}{|c|}{ PLCR-3 } \\
\hline & EM & $\mathbf{G}$ & EM & G & EM & G \\
\hline $1: 1$ & 0.0 & 0.0 & 0.0 & $7.0 \pm 0.6^{a}$ & $5.0 \pm 0.6^{a}$ & 0.0 \\
\hline $1: 2$ & 0.0 & 0.0 & $5.0 \pm 0.6^{a}$ & 0.0 & 0.0 & 0.0 \\
\hline $1: 3$ & 0.0 & 0.0 & 0.0 & 0.0 & 0.0 & 0.0 \\
\hline $1: 4$ & 0.0 & 0.0 & 0.0 & 0.0 & 0.0 & 0.0 \\
\hline $1: 5$ & 0.0 & 0.0 & 0.0 & 0.0 & 0.0 & 0.0 \\
\hline
\end{tabular}

Cada valor es la media \pm desviación estándar, $n=3$. Literales o letras diferentes indican diferencias significativas $(p<0.05)$.

considerando que este estudio es el pionero en la adición de chalconas sintetizadas a extractos etanólicos de propóleos para incrementar su actividad antifúngica y su utilización como tratamiento complementario para infecciones por hongos. Por lo tanto, es también importante realizar el estudio con cepas de aislamientos clínicos para ambos hongos con el fin de evaluar la resistencia antifúngica a nuevos compuestos.

\section{CONCLUSIONES}

La actividad antifúngica de los propóleos esta determinada por la cantidad de compuestos fenólicos, cuando estos metabolitos se encuentran en bajas cantidades o ausentes, la actividad es baja o nula; pero con la adición de precursores de flavonoides denominados chalconas, se observó la potencialización del efecto antifúngico. La chalcona sintética que presentó en su estructura química mayor número de sustituyentes hidroxilo en los anillos $\mathrm{A}$ y $B$, denominada PLCR-2, mejoró la potencialización del efecto frente a Candida albicans y Aspergillus flavus, actuando de diferente forma con base a la proporción utilizada; por lo anterior, es necesario ampliar la investigación del mecanismo de acción de la relación chalcona:propóleo para validar estos resultados y finalmente, esta investigación brinda apoyo al apicultor que utiliza esta resina como materia prima para la elaboración de productos.

\section{AGRADECIMIENTOS}

Los autores agradecen el apoyo recibido de los proyectos: UNAM-DGAPA, PAPIIT IT 200915, PAPIIT IN217118, FESC:PIAPIC 1632, PIAPI 1802, IN223719. Betsabé Rodríguez Pérez es estudiante de doctorado en el Programa de Producción y Salud Animal de la Universidad Nacional Autónoma de México (UNAM) con beca del Consejo Nacional de Ciencia y Tecnología, México (Conacyt) (No. 511917).

\section{REFERENCIAS}

Al, N.Y., Sun, J., Robertson, A., Giesy, J. P. y Wiseman, S. 2016. Chemical characterization and antioxidant properties of Canadian propolis. Journal Apicultural Research. 55(4):305314.

Bader, O., Tünnermann, J., Dudakova, A. y Tangwattanachuleeporn, M. 2015. Environmental azole resistant Aspergillus fumigatus in Germany. Antimicrobial Agents and Chemotherapy. 59: 4356-4359.

Bankova, V. 2005. Chemical diversity of propolis and the problem of standardization. Journal of Ethnopharmacology. 100(2005):114-117.

Bankova, V. 2009. Chemical diversity of propolis makes it a valuable source of new biologically active compounds. Journal of Apiculture Product and Apiculture Medical Science. 1(2):23-28.

Bankova, V., Bertelli, D., Borba, R., Conti, B., Barbosa da-Silva, I., Danert, C., Nogueira, E., Falcão, S., Isla, M., Nieva, M., Papotti, G., Popova, M., Basso, S. K., Salas, A., Frankland, S. A., Vilczaki, S., Sforcin, J., Simone-Finstrom, M., Spivak, M., Trusheva, B., Vilas-Boas, M., Wilson, M. y Zampini, C. 2016. Standard methods for Apis mellifera propolis research. Journal of Apicultural Research. 56(SI3): 1-49.

Blanco, P. M., Dip, G. A., Torres, R. E., Peña, F. D., Vogel, C., Bullaín, G. M. y Fajardo, C. L. 2016. Actividad antibacteriana in vitro de nuevas chalconas halogenadas. Química Viva. 15(2):17-28.

Bucio, V, C., Navarro, L. F., Martínez, J. O., Torres, M. J. y García, A. E. 2016. Producción de propóleo en campo, recolectado por las abejas productoras de miel (Apis mellifera). Investigación y Desarrollo en Ciencia y Tecnología de Alimentos. 1(1): 515-520.

Chetana, B. P., Mahajan, S. K. y Suvarna, A. K. 2009. Chalcone: A Versatile Molecule. Journal of Pharmaceutical Sciences and Research. 1(3):11-22.

Clinical and Laboratory Standards Institute (CLSI/M44-A2), Method for Antifungal Disk Diffusion Susceptibility Testing of Yeasts [Consultado 26 febrero 2018] 2009. Disponible en: https://clsi.org/media/1634/m44a2_sample.pdf.

Cruz, S. T. A., Estrada, G. P. A., López, Z. C. I., Autrán, M. M., Pérez, V. V. y Londoño, O. A. 2014. Use of Propolis for Topical Treatment of Dermatophytosis in Dog. Open Journal of Veterinary Medicine. 4: 239-245.

Díaz-Tielas, C., Graña, E., Reigosa, M. J. y Sánchez-Moreira, A. 2016. Biological activities and novel applications of chalcones. Planta Daninha. 34(3):607-616.

Flores, I., Moreno, M., Londoño, A. y Cruz, T. 2016. Use of Mexican Propolis for the Topical Treatment of Dermatomycosis in Horses. Open Journal of Veterinary Medicine. 6: 1-8.

García-Andrade, M., González-Laredo, R., Rocha-Guzmán, N. y Gallegos-Infante, J. 2013. Mesquite leaves (Prosopis laevigata), a natural resource with antioxidant capacity and 
cardioprotection potential. Industrial Crops and Products. 44(2013):336-342.

González-Búrquez, M. J., González-Díaz, R. F., García-Tovar, C. G., Carrillo-Miranda, L., Soto-Zarate, C. I., CanalesRamírez, M. M., Penieres-Carrillo, J. G., Cruz-Sánchez, T. A. y Fonseca-Coronado, S. 2018. Comparison between In Vitro Antiviral Effect of Mexican Propolis and Three Commercial Flavonoids against Canine Distemper Virus. Evidence-Based Complementary and Alternative Medicine. 2018(7092416):1-9.

Guerrero, V. L. 2016. Estudio de la reactividad de chalconas como precursores en la síntesis de nuevos compuestos pirazolínicos, betalactámicos y tiazolidínicos fusionados. Maestra en Ciencias. Tesis, Universidad Nacional de Colombia, Bogotá, Colombia.

Hernández-Zarate, M., Abraham-Juárez, M., Cerón-García, A., Gutiérrez-Chávez, D., Gutiérrez-Arenas, D. y Ávila-Ramos, F. 2017. Contenido de flavonoides, fenoles y actividad antioxidante de propóleos colectados en Guanajuato, México. Investigación y Desarrollo en Ciencia y Tecnología de Alimentos. 2(2017): 607-612.

Hormaza, A. A., Arbeláez, O. F. y Montes, V. N. 2009. Síntesis y caracterización espectroscópica de una serie de chalconas. Scientia et Technica. XV (43):316-320.

Joya, M., Gil, M. y Bastidas-Pacheco, G. 2017. Actividad fungistática y fungicida de extractos etanólicos de propóleos sobre el crecimiento in vitro de cepas del género Candida. Tecnología en Marcha. 30(3):3-11.

Kulkarni, G. B., Siva, R. A., Padmavathi, A. y Raamadevi, K. 2009. 3D-QSAR Studies of anti-bacterial chalcones. International Journal of ChemTech Research. 1(3):717-721.

Larone, D.H. 2011. Medically Important Fungi: A Guide to Identification. ASM Press, Washington, D.C.

Londoño, A. O. 2010. Estudio comparativo de la actividad antifúngica de un extracto de propóleo mexicano y de tres plantas que Apis mellifera usa para su producción. Doctora en Ciencias. Tesis, Instituto Tecnológico de Costa Rica, Costa Rica.

Londoño-Orozco, A., Penieres-Carrillo, J. G., García-Továr, C. G., Carrillo-Miranda, L., Quintero-Mora, M. L., García-Vásquez, S. E., Mendoza-Saavedra, M. A. y Cruz-Sánchez, T. A. 2008. Estudio de la actividad antifúngica de un extracto de propóleo de la abeja Apis mellifera proveniente del Estado de México. Tecnología en Marcha. 21(1):49-55.

López, N. S., Castelli, V. M., Zacchino, A. S., Domínguez, N. J., Lobo, G., Charris-Charris, J., Cortés, G. J., Ribas, C. J., Devia, C., Rodríguez, A. M. y Enriz, D. R. 2001. In Vitro Antifungal Evaluation and Structure-Activity Relationships of a New Series of Chalcone Derivatives and Synthetic Analogues, with Inhibitory Properties Against Polymers of the Fungal Cell Wall. Bioorganic \& Medicinal Chemistry. 9:1999-2013.

Lotti, C., Campo, M., Piccinelli, A., Cuesta-Rubio, O., Márquez, I. y Rastrelli, L. 2010. Chemical Constituents of Red Mexican Propolis. Journal of Agriculture and Food Chemistry. 58:2209 $-2213$.

Lozina, L., Peichoto, M. E., Acosta, O. y Granero, G. E. 2010. Estandarización y Caracterización Organoléptica y FísicoQuímica de 15 Propóleos Argentinos. Latin American Journal of Pharmacy. 29(1):102-110.

Lugo, E., Goycolea, V. F., Hernández, J. y Velásquez, C. 2006. Pinocembrina, principal componente de propóleos sonorenses. Invurnus. 1(2):71-83.
Mahapatra, D. K. y Bharti, S. K. 2016. Therapeutic potential of chalcones as cardiovascular agents. Life Sciences. 148 (2016):154-172.

Mellado, E., Cuenca-Estrella, M. y Rodríguez-Tudela, J. L. 2002. Importancia clínica de los mecanismos de resistencia de los hongos filamentosos a los antifúngicos. Enfermedades Infecciosas y Microbiología Clínica. 20(10):523-530.

Miguel, M. G, Nunes, S., Dandle, S. A., Cavaco, A. M. y Antunes, M. D. 2014. Phenols, flavonoids and antioxidant activity of aqueous and methanolic extracts of propolis (Apis mellifera L.) from Algarve, South Portugal. Food Science and Technology. 34(1):16-23.

Mirzoeva, K., Grishanin, N. y Calder P. 1997. Antimicrobial action of propolis and some of its components: the effects on growth, membrane potential and motility of bacteria. Microbiological Research. 152(1997):239-246.

Norma Oficial Mexicana NOM-003-SAG/GAN-2017, Propóleos, producción y especificaciones para su procesamiento. [Consultado 6 octubre 2017] 2017. Disponible en: https:// dof.vlex.com.mx/c/Norma-Oficial-Mexicana.

Pfaller, M. 2012. Antifungal drug resistance: mechanisms, epidemiology, and consequences for treatment. The American Journal of Medicine. 125(1): S3-13.

Quintero-Mora, M. L., Londoño-Orozco, A., HernándezHernández, F., Manzano-Gayoso, P., López-Martínez, R., Soto-Zarate, C. I., Carrillo-Miranda, L., Penieres-Carrillo, J. G., García-Továr, C. G. y Cruz-Sánchez, T. A. 2008. Efecto de extractos de propóleos mexicanos de Apis mellifera sobre el crecimiento in vitro de Candida albicans. Revista Iberoamericana de Micología. 25:22-26.

Quintero-Mora, M. L., Londoño-Orozco, A., Soto-Zarate, C. I., García-Tovar, C. G. Carrillo-Miranda, L., Penieres-Carrillo, J. G., y Cruz-Sánchez, T. A. 2011. Structural and genetic alterations of fungal cells caused by Mexican propolis. Science against microbial pathogens: communicating current research and technological advances (FORMATEX). 1068-1072.

Ramírez, E. M., Barajas, B. L., Pérez, B. C., Sáenz, G. A. y Silva, B. S. 2012. Síntesis y actividad biológica de chalconas. Revista Mexicana de Ciencias Farmacéuticas. 43(4): 7-14.

Rasul, A., Millimouno, F., Eltayb, W., Ali, M., Li, J. y Li, X. 2013. Pinocembrin: A Novel Natural Compound with Versatile Pharmacological and Biological Activities. BioMed Research International. 2013(379850):1-9.

Rivera-Yáñez, N., Rodríguez-Canales, M., Nieto-Yáñez, O., Jiménez-Estrada, M., Ibarra-Barajas, M., Canales-Martínez, M. M. y Rodríguez-Monroy, M. A. 2018. Hypoglycaemic and Antioxidant Effects of Propolis of Chihuahua in a Model of Experimental Diabetes. Evidence-Based Complementary and Alternative Medicine. 2018(4360356):1-10.

Rivera-Yáñez, C. R., Terrazas, L I., Jiménez-Estrada, M., Campos, J. E., Flores-Ortiz, C. M., Hernández, L. B., Cruz-Sánchez, T. A., Garrido-Fariña, G. I., Rodríguez-Monroy, M. A. y CanalesMartínez, M. M. 2017. Anti-Candida Activity of Bursera morelensis Ramirez Essential Oil and Two Compounds, a-Pinene and $\gamma$-Terpinene, An In Vitro Study. Molecules. 22(12):1-13.

Rodríguez, R. B. 2015. Perfil químico de propóleos mexicanos para su aplicación en medicina veterinaria. Maestría en Ciencias, Tesis. Universidad Nacional Autónoma de México. Estado de México, México. 
Sapra, S., Sharma, K., Bhalla, Y. y Dhar, K. L. 2016. Chalconoid Derived Heterocycles as Potent Bioactive Molecules: A Review. Chemical Sciences Journal. 7(2):3-8.

Silva-Carvalho, R., Baltazar, F. y Almeida-Aguiar, C. 2015. Propolis: A Complex Natural Product with a Plethora of Biological Activities That Can Be Explored for Drug Development. Evidence-Based Complementary and Alternative Medicine. 2015(206439):1-29.

Singh, P., Anand, A. y Kumar, V. 2014. Recent developments in biological activities of chalcones: A mini review. European Journal of Medicinal Chemistry. 85:758-777.

Sivakumar, P. M., Muthu, K. T. y Doble, M. 2009. Antifungal activity, mechanism and QSAR studies on chalcones. Chemical Biology \& Drug Design. 74(1):68-79.

Sosa-López, Á. A., Cabrera, M. G. y Álvarez, M. Y. 2017. Parámetros físicos y características organolépticas de propóleos provenientes de la Provincia de Misiones, Argentina. Journal of the Selva Andina Biosphere. 5(1):51-58.
Stepanović, S., Antić, N., Dakić, I. y Švabić-Vlahović, M. 2003. Antimicrobial activity of propolis and synergism between propolis and antimicrobial drugs. Microbiological Research. 158: 353-357.

Tapia, C. 2005. Mecanismos de acción, reacciones adversas y nuevos antimicóticos. Programa de Microbiología y Micología. ICBM. Facultad de Medicina, Universidad de Chile.

Trusheva, B., Trunkova, D. y Bankova, V. 2007. Different extraction methods of biologically active components from propolis: a preliminary study. Chemical Central Journal. 1(13):1-13.

Vargas-Sánchez, R. D, Torrescano-Urrutia, G. R., Mendoza-Wilson, A. M., Vallejo-Galland, B., Acedo-Félix, E., Sánchez-Escalante, J. J., Peñalba-Garmendia, M. C. y Sánchez-Escalante, A. 2014. Mecanismos involucrados en la actividad antioxidante y antibacteriana del propóleos. Biotecnia. XVI (1):32-37.

Viloria, B. J., Gil, G. H., Durango, R. D. y García, P. C. 2012. Caracterización fisicoquímica del propóleo de la región del bajo cauca antioqueño (Antioquia, Colombia). Biotecnología en el Sector Agropecuario y Agroindustrial. 10(1):77-86. 